\title{
New Perspectives on Classical Meanness of Some Ladder Graphs
}

\author{
A. M. Alanazi $\mathbb{D}^{1},{ }^{1}$ G. Muhiuddin $\mathbb{D}^{1},{ }^{1}$ A. R. Kannan, ${ }^{2}$ and V. Govindan ${ }^{3}$ \\ ${ }^{1}$ Department of Mathematics, University of Tabuk, Tabuk 71491, Saudi Arabia \\ ${ }^{2}$ Department of Mathematics, Mepco Schlenk Engineering College, Sivakasi 626 005, Tamil Nadu, India \\ ${ }^{3}$ Department of Mathematics, Sri Vidya Mandir Arts \& Science College, Katteri, Uthangarai 636902, Tamilnadu, India
}

Correspondence should be addressed to G. Muhiuddin; chishtygm@gmail.com

Received 3 April 2021; Accepted 14 June 2021; Published 30 June 2021

Academic Editor: A. H. Kara

Copyright $\odot 2021$ A. M. Alanazi et al. This is an open access article distributed under the Creative Commons Attribution License, which permits unrestricted use, distribution, and reproduction in any medium, provided the original work is properly cited.

In this study, we investigate a new kind of mean labeling of graph. The ladder graph plays an important role in the area of communication networks, coding theory, and transportation engineering. Also, we found interesting new results corresponding to classical mean labeling for some ladder-related graphs and corona of ladder graphs with suitable examples.

\section{Introduction and Preliminaries}

All through this paper, by a graph, we mean an undirected, simple, and finite graph. For documentations and phrasing, we follow [1-6]. For a point-by-point review on graph labeling, refer [7].

Let $P_{n}$ be a path on $n$ nodes denoted by $u_{1, \gamma}$, where $1 \leq \gamma \leq n$, and with $n-1$ lines denoted by $e_{1, \delta}$, where $1 \leq \delta \leq n-1$, where $e_{\gamma}$ is the line joining the vertices $u_{1, \gamma}$ and $u_{1, \gamma+1}$. On each edge $e_{\delta}$, erect a ladder with $n-(\gamma-1)$ steps including the edge $e_{\gamma}$, for $\gamma=1,2,3, \ldots, n-1$. The resulting graph is called the one-sided step graph, and it is denoted by $\mathrm{ST}_{n}$. Let $P_{2 n}$ be a path on $2 n$ vertices $u_{1, \gamma}$, where $1 \leq \gamma \leq 2 n$ and with $2 n-1$ edges $e_{1}, e_{2}, \ldots, e_{2 n-1}$, where $e_{\gamma}$ is the line joining the vertices $u_{1, \gamma}$ and $u_{1, \gamma+1}$. On each edge $e_{\gamma}$, we erect a ladder with ' $\gamma+1$ ' steps including the edge $e_{\gamma}$, for $\gamma=1,2,3, \ldots, n$, and on each $e_{\gamma}$, we erect a ladder with $2 n+$ $1-\gamma$ steps including $e_{\gamma}$, for $\gamma=n+1, n+2, \ldots, 2 n-1$. The graph thus obtained is called the double-sided step graph, and it is denoted by $2 \mathrm{ST}_{2 n}$. Let $G_{1}$ and $G_{2}$ be any two graphs with $p_{1}$ and $p_{2}$ vertices, respectively. Then, $G_{1} \times G_{2}$ is the cartesian product of two graphs. A ladder graph $L_{n}$ is the graph $P_{2} \times P_{n}$. The graph $G \circ S_{m}$ is obtained from $G$ by attaching $m$ pendant vertices to each vertex of $G$. The triangular ladder $\mathrm{TL}_{n}$, for $n \geq 2$, is a graph obtained from two paths by $u_{1}, u_{2}, \ldots u_{n}$ and $v_{1}, v_{2}, \ldots v_{n}$ by adding the edges $u_{\gamma} v_{\gamma}, 1 \leq \gamma \leq n$ and $u_{\gamma} v_{\gamma+1}, 1 \leq \gamma \leq n-1$. The slanting ladder $\mathrm{SL}_{n}$ is a graph obtained from two paths $u_{1}, u_{2}, \ldots u_{n}$ and $v_{1}, v_{2}, \ldots v_{n}$ by joining each $v_{\gamma}$, with $u_{\gamma+1}, 1 \leq \gamma \leq n-1$. The graph $D_{n}^{*}$ having the vertices $\left\{a_{\gamma, \delta}: 1 \leq \gamma \leq n, \delta=1,2,3,4\right\}$, and its edge set is $\left\{a_{\gamma, 1} a_{\gamma+1,1}, a_{\gamma, 3} a_{\gamma+1,3}: 1 \leq \gamma \leq n-1\right\}$ $\cup\left\{a_{\gamma, 1} a_{\gamma, 2}, a_{\gamma, 2} a_{\gamma, 3}, a_{\gamma, 3} a_{\gamma, 4}, a_{\gamma, 4} a_{\gamma, 1}: 1 \leq \gamma \leq n\right\}$.

\section{Literature Survey}

The origin of graph labeling called graceful labeling was characterized by Rosa in [8] and the mean labeling of graphs was introduced by Somasundram et al. in [9]. In [10], Arockiaraj et al. presented the idea of $F$-root square mean labeling of the graphs and examined its meanness [11]. Durai Baskar and Arockiaraj talked about the C-geometric meanness of some ladders in [12]. Dafik et al. researched the antimagicness of the graphs including the graph $D_{n}^{*}$ in [13]. Durai Baskar considered the logarithmic meanness in [14] and Rajesh Kannan et al. characterized idea of exponential mean graphs in [15]. In addition, more concepts of ladder graphs and related concepts have been studied in [16-24]. Recently, Muhiuddin et al. have applied various related concepts on graphs in different aspects (see, e.g., [25-31]).

\section{Methodology}

A labeling $\chi$ on a graph $G(V, E)$ with $p$ vertices and $q$ edges is called a Smarandache $(m, k)$ mean labeling, for an integer $m \geq 1$ and $k \geq 2$, if $\chi: V(G) \longrightarrow\{1,2,3, \ldots, q+1\}$ is injective 


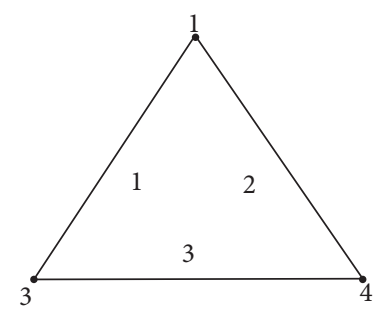

Figure 1: A classical mean labeling of $C_{3}$.

and the induced function $\chi^{*}: E(G) \longrightarrow\{1,2,3, \ldots, q\}$ de-

fined by

$$
\chi^{*}(u v)=\left\lfloor\frac{1}{4}\left(\sqrt[m]{\frac{\chi(u)^{m}+\chi(v)^{m}}{2}}+\sqrt[k]{\chi(u)^{m} \chi(v)^{m}}+\frac{2 \chi(u)^{m} \chi(v)^{m}}{\chi(u)^{m}+\chi(v)^{m}}+\sqrt[k]{\frac{\chi(u)^{k}+\chi(v)^{k}}{2}}\right)\right\rfloor,
$$

for all $u v \in E(G)$, is bijective.

Particularly, if $m=1$ and $k=2$, such a Smarandache $(m, k)$ mean labeling is the classical mean labeling on the graph. A function $\chi$ is known as a classical mean labeling of a graph $G(V, E)$ with $p$ nodes and $q$ edges if $\chi: V(G) \longrightarrow\{1,2,3, \ldots, q+1\}$ is injective and the incited edge assignment function $\chi^{*}: E(G) \longrightarrow\{1,2,3, \ldots, q\}$ characterized as

$$
\chi^{*}(u v)=\left\lfloor\frac{1}{4}\left(\frac{\chi(u)+\chi(v)}{2}+\sqrt{\chi(u) \chi(v)}+\frac{2 \chi(u) \chi(v)}{\chi(u)+\chi(v)}+\sqrt{\frac{\chi(u)^{2}+\chi(v)^{2}}{2}}\right)\right\rfloor
$$

for all $u v \in E(G)$, is bijective. A graph that concedes a classical mean labeling is said to be classical mean graph.

A classical mean labeling of $C_{3}$ is shown in Figure 1.

Here, we found interesting new results corresponding to classical mean labeling for some ladder-related graphs and corona of ladder graphs.

\section{Main Results}

Theorem 1. The one-sided step graph $S T_{n}$ is a classical mean graph, for $n \geq 2$.

Proof. Let $P_{n}$ be a path on $n$ nodes denoted by $u_{1, \gamma}$, where $1 \leq \gamma \leq n$ and with $n-1$ lines denoted by $e_{1, \delta}$, where $1 \leq \delta \leq n-1$, where $e_{\gamma}$ is the line joining the vertices $u_{1, \gamma}$ and $u_{1, \gamma+1}$. On each edge $e_{\delta}$, erect a ladder with $n-(\gamma-1)$ steps including the edge $e_{\gamma}$, for $\gamma=1,2,3, \ldots, n-1$. The resulting graph is called the one-sided step graph, and it is denoted by $\mathrm{ST}_{n}$. Let $u_{1, a}, u_{2, a}, u_{3, b}, u_{4, c}, \ldots, u_{n, 1}, \quad u_{n, 2}$, for $1 \leq a \leq n$, $1 \leq b \leq n-1$, and $1 \leq c \leq n-2$, be the nodes of $\mathrm{ST}_{n}$.

Construct a mapping $\chi$ from $V(G)$ to $\left\{1,2,3, \ldots,-1+n+n^{2}\right\}$ :

$\chi\left(u_{\gamma, \delta}\right)=(1-\gamma+n)^{2}+\delta-1, \quad$ for $2 \leq \gamma \leq n, 1 \leq \delta \leq n+2-\gamma$,

$\chi\left(u_{1, \delta}\right)=n^{2}+\delta-1, \quad$ for $2 \leq \delta \leq n$.

Therefore,

$$
\begin{aligned}
& \chi^{*}\left(u_{\gamma, \delta} u_{\gamma, \delta+1}\right)=(1-\gamma+n)^{2}+\delta-1, \quad \text { for } 2 \leq \gamma \leq n \text { and } 1 \leq \delta \leq 1-\gamma+n, \\
& \chi^{*}\left(u_{1, \delta} u_{1, \delta+1}\right)=-1+n^{2}+\delta, \quad 1 \leq \delta \leq n-1, \\
& \chi^{*}\left(u_{\gamma, \delta} u_{\gamma+1, \delta}\right)=(1-\gamma+n)(-\gamma+n)-1+\delta, \quad \text { for } 1 \leq \gamma \leq-1+n \text { and } 1 \leq \delta \leq n+1-\gamma .
\end{aligned}
$$

Hence, one-sided step graph $\mathrm{ST}_{n}$ is a classical mean graph, for $n \geq 2$.
A classical mean labeling of $\mathrm{ST}_{7}$ is shown in Figure 2. 
Theorem 2. The double-sided step graph $2 S T_{2 n}$ is a classical mean graph, for $n \geq 1$.

Proof. Let $P_{2 n}$ be a path on $2 n$ vertices $u_{1, \gamma}$, where $1 \leq \gamma \leq 2 n$ and with $2 n-1$ edges $e_{1}, e_{2}, \ldots, e_{2 n-1}$, where $e_{\gamma}$ is the line joining the vertices $u_{1, \gamma}$ and $u_{1, \gamma+1}$. On each edge $e_{\gamma}$, we erect a ladder with ' $\gamma+1^{\text {' }}$ steps including the edge $e_{\gamma}$, for $\gamma=1,2,3, \ldots, n$, and on each $e_{\gamma}$, we erect a ladder with $2 n+$ $1-\gamma$ steps including $e_{\gamma}$, for $\gamma=n+1, n+2, \ldots, 2 n-1$. The graph thus obtained is called the double-sided step graph, and it is denoted by $2 \mathrm{ST}_{2 n}$.

Let $u_{1, a}, u_{2, a}, u_{3, b}, u_{4, c}, \ldots, u_{n+1,1}, u_{n+1,2}, \quad$ for $\quad 1 \leq a \leq$ $2 n, 1 \leq b \leq 2 n-2$, and $1 \leq c \leq 2 n-4$ be the nodes of $2 \mathrm{ST}_{2 n}$.

Construct a mapping $\chi$ from $V(G)$ to $\left\{1,2,3, \ldots, 3 n+2 n^{2}\right\}$ :

$$
\chi\left(u_{1, \delta}\right)= \begin{cases}1+n+2 n^{2}+2(-1+\delta), & 1 \leq \delta \leq n, \\ 3 n+2 n^{2}-2 \delta+2 n+2, & 1+n \leq \delta \leq 2 n,\end{cases}
$$

for $2 \leq \delta \leq 2+n-\gamma$ and $2 \leq \gamma \leq n$,

$$
\chi\left(u_{\gamma, \delta}\right)=2(1-\gamma+n)^{2}+(n+2-\gamma)+2(\delta-2),
$$

for $2 \leq \gamma \leq n$ and $n+3-\gamma \leq \delta \leq 2 n+3-2 \gamma$,

$$
\begin{aligned}
\chi\left(u_{\gamma, \delta}\right) & =2(1+n-\gamma)^{2}+3(n+1-\gamma)-2(\gamma+\delta-n-3), \\
\chi\left(u_{2,1}\right) & =2 n^{2}+n-2, \\
\chi\left(u_{\gamma, 1}\right) & =2(2+n-\gamma)^{2}-\gamma+n, \quad 3 \leq \gamma \leq n+1, \\
\chi\left(u_{\gamma, 2 n+4-2 \gamma}\right) & =2(n+2-\gamma)^{2}+1+n-\gamma, \quad 2 \leq \gamma \leq n+1 .
\end{aligned}
$$

Therefore,

$$
\chi^{*}\left(u_{1, \delta} u_{1, \delta+1}\right)= \begin{cases}1+n+2 n^{2}+2(-1+\delta), & 1 \leq \delta \leq n, \\ 3 n+2 n^{2}-2 \delta+2 n, & n+1 \leq \delta \leq 2 n-1,\end{cases}
$$

for $2 \leq \delta \leq 2+n-\gamma$ and $2 \leq \gamma \leq n-1$,

$$
\chi^{*}\left(u_{\gamma, \delta} u_{\gamma, \delta+1}\right)=2(-\gamma+1+n)^{2}+(-\gamma+n+2)+2(-2+\delta) \text {, }
$$

for $-\gamma+3+n \leq \delta \leq 2+2 n-2 \gamma$ and $2 \leq \gamma \leq n-1$,

$$
\begin{aligned}
& \chi^{*}\left(u_{\gamma, \delta} u_{\gamma, \delta+1}\right)=2(-\gamma+n+1)^{2}+3(-\gamma+n+1)-2(-2+\gamma+\delta-n), \\
& \chi^{*}\left(u_{\gamma, 2 n+3-2 \gamma} u_{\gamma+1,2 n+2-2 \gamma}\right)=2(-\gamma+n+1)^{2}+(-\gamma+n+1), \quad \text { for } 2 \leq \gamma \leq n \text {, } \\
& \chi^{*}\left(u_{n, 2} u_{n, 3}\right)=4 \text {, } \\
& \chi^{*}\left(u_{n+1,1} u_{n+1,2}\right)=1 \text {, } \\
& \chi^{*}\left(u_{1,1} u_{2,1}\right)=2 n^{2}+n-1, \\
& \chi^{*}\left(u_{1,2 n} u_{2,2 n}\right)=2 n^{2}+n, \\
& \chi^{*}\left(u_{\gamma, 2} u_{\gamma+1,1}\right)=2(-\gamma+n)^{2}-\gamma+n, \quad 2 \leq \gamma \leq n, \\
& \chi^{*}\left(u_{1, \delta} u_{2, \delta}\right)= \begin{cases}1-n+2 n^{2}+2 \delta-4, & 2 \leq \delta \leq n, \\
-2+n+2 n^{2}-2(-1-n+\delta), & 1+n \leq \delta \leq-1+2 n,\end{cases}
\end{aligned}
$$

for $3 \leq \delta \leq n+2-\gamma$ and $2 \leq \gamma \leq n-1$,

for $3+n-\gamma \leq \delta \leq 2+2 n-2 \gamma$ and $2 \leq \gamma \leq n-1$,

$$
\chi^{*}\left(u_{\gamma, \delta} u_{\gamma+1, \delta-1}\right)=2(-\gamma+1+n)^{2}-(-\gamma+2+n)+2 \delta-4,
$$

$$
\begin{aligned}
\chi^{*}\left(u_{\gamma, \delta} u_{\gamma+1, \delta-1}\right) & =2(-\gamma+1+n)^{2}+(-\gamma+3+n)-2 \gamma-2 \delta+2 n+2, \\
\chi^{*}\left(u_{\gamma, 1} u_{\gamma, 2}\right) & =2(-\gamma+1+n+1)^{2}+3(-\gamma+1+n), \quad \text { for } 2 \leq \gamma \leq n, \\
\chi^{*}\left(u_{\gamma, 2 n+3-2 \gamma} u_{\gamma, 2 n+4-2 \gamma}\right) & =2(-\gamma+1+n)^{2}+3(-\gamma+1+n)+1, \quad \text { for } 2 \leq \gamma \leq n .
\end{aligned}
$$

Hence, the double-sided step graph $2 \mathrm{ST}_{2 n}$ is a classical mean graph, for $n \geq 1$.
A classical mean labeling of $2 \mathrm{ST}_{10}$ is shown in Figure 3. 


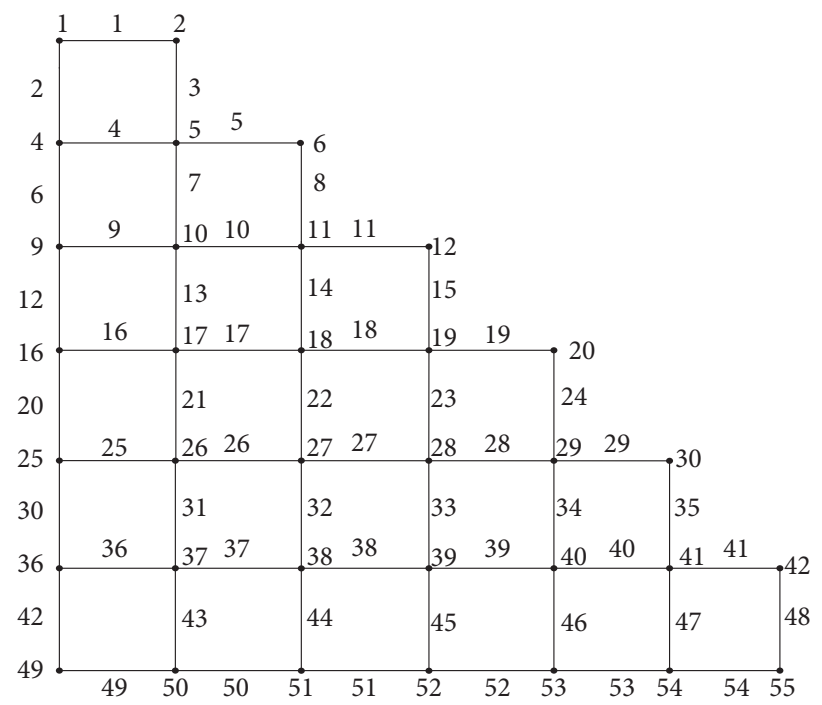

FIgURE 2: A classical mean labeling of $\mathrm{ST}_{7}$.

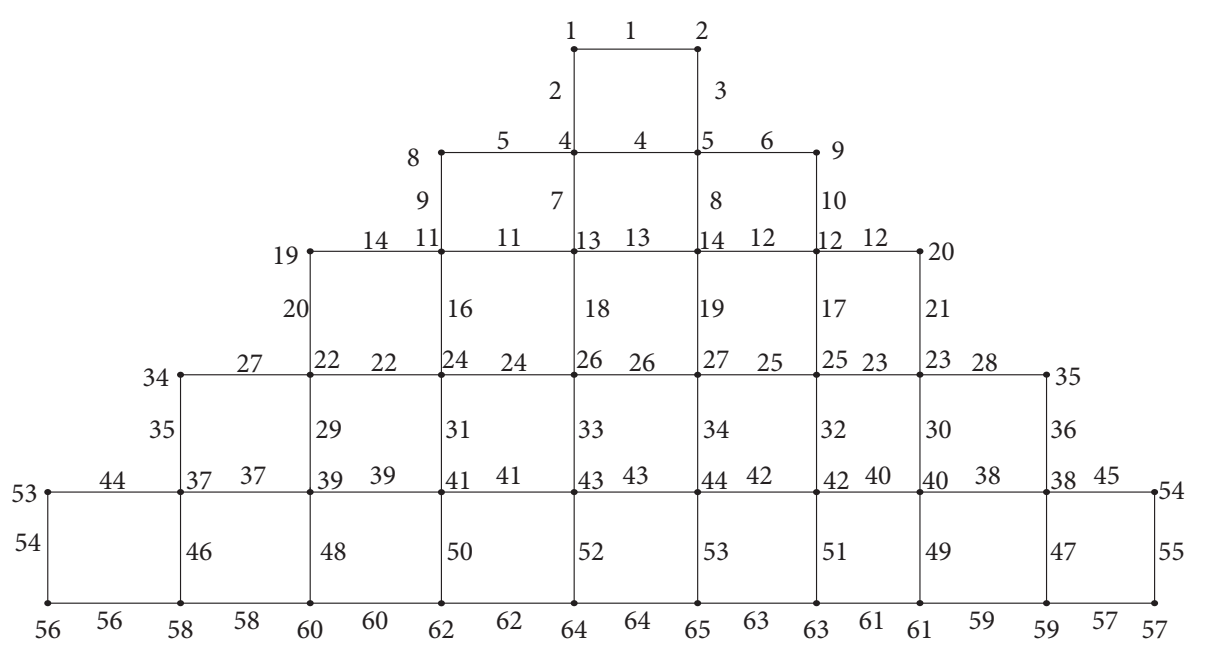

Figure 3: A classical mean labeling of $2 \mathrm{ST}_{10}$.

Theorem 3. For $n \geq 2$ and $m \leq 4$, the graph $P_{m} \times P_{n}$ is a classical mean graph.

Proof. Take $V\left(P_{m} \times P_{n}\right)=\left\{v_{\gamma \delta}: 1 \leq \delta \leq n, 1 \leq \gamma \leq m\right\}$ and $E\left(P_{m} \times P_{n}\right)=\left\{v_{\gamma \delta} v_{(\gamma+1) \delta}: \quad 1 \leq \delta \leq n, 1 \leq \gamma \leq m-1\right\} \cup\left\{v_{\gamma \delta}\right.$ $\left.v_{\gamma(\delta+1)}: 1 \leq \delta \leq n-1,1 \leq \gamma \leq m\right\}$.

Case (i). $m=2$.

Construct a mapping $\chi$ from $\left.V\left(P_{2} \times P_{n}\right)\right)$ to $\{1,2,3, \ldots, 3 n-1\}$ :

$$
\chi\left(v_{\gamma \delta}\right)=\gamma+3(\delta-1), \quad \text { for } 1 \leq \gamma \leq 2 \text { and } 1 \leq \delta \leq n .
$$

Therefore,

$$
\begin{aligned}
\chi^{*}\left(v_{1 \delta} v_{2 \delta}\right) & =3 \delta-2, \quad \text { for } 1 \leq \delta \leq n, \\
\chi^{*}\left(v_{\gamma \delta} v_{\gamma(\delta+1)}\right) & =\gamma+3 \delta-2, \quad \text { for } 1 \leq \gamma \leq 2 \text { and } 1 \leq \delta \leq n-1 .
\end{aligned}
$$

Case (ii). $m=3$.

Construct a mapping $\chi$ from $V\left(P_{3} \times P_{n}\right)$ to $\{1,2,3, \ldots, 5 n-2\}$ :

$$
\begin{aligned}
& \chi\left(v_{\gamma 1}\right)= \begin{cases}\gamma, & 1 \leq \gamma \leq 2, \\
4, & \gamma=3,\end{cases} \\
& \chi\left(v_{\gamma \delta}\right)=\gamma+5(\delta-1), \quad \text { for } 1 \leq \gamma \leq 3 \text { and } 2 \leq \delta \leq n .
\end{aligned}
$$

Therefore, 


$$
\begin{aligned}
\chi^{*}\left(v_{\gamma 1} v_{(\gamma+1) 1}\right) & =\gamma, \quad \text { for } 1 \leq \gamma \leq 2, \\
\chi^{*}\left(v_{\gamma 1} v_{\gamma 2}\right) & =\gamma+2, \quad \text { for } 1 \leq \gamma \leq 3, \\
\chi^{*}\left(v_{\gamma \delta} v_{(\gamma+1) \delta}\right) & =\gamma+5 \delta-5, \quad \text { for } 1 \leq \gamma \leq 2 \text { and } 2 \leq \delta \leq n, \\
\chi^{*}\left(v_{\gamma \delta} v_{\gamma(\delta+1)}\right) & =\gamma+5 \delta-3, \quad \text { for } 1 \leq \gamma \leq 3 \text { and } 2 \leq \delta \leq n-1 .
\end{aligned}
$$

Case (iii). $m=4$.

Consider the graph $P_{4} \times P_{n}$, for $n \geq 3$.

Construct a mapping $\chi$ from $V\left(P_{4} \times P_{n}\right)$ to $\{1,2,3, \ldots, 7 n-3\}$ :

$\chi\left(v_{\gamma 1}\right)= \begin{cases}\gamma, & 1 \leq \gamma \leq 2, \\ \gamma+1, & 3 \leq \gamma \leq 4,\end{cases}$

$\chi\left(v_{\gamma 2}\right)=\gamma+7, \quad$ for $1 \leq \gamma \leq 4$,

$\chi\left(v_{\gamma \delta}\right)=\gamma+7(\delta-1), \quad$ for $1 \leq \gamma \leq 4$ and $3 \leq \delta \leq n$.

Therefore,

$\chi^{*}\left(v_{1 \delta} v_{1(\delta+1)}\right)= \begin{cases}8 \delta-5, & 1 \leq \delta \leq 2, \\ 7 \delta-3, & 3 \leq \delta \leq n-1,\end{cases}$

$\chi^{*}\left(v_{\gamma \delta} v_{\gamma(\delta+1)}\right)=\gamma+7 \delta-4, \quad$ for $2 \leq \gamma \leq 4$ and $1 \leq \delta \leq n-1$,

$\chi^{*}\left(v_{\gamma 1} v_{(\gamma+1) 1}\right)= \begin{cases}\gamma, & 1 \leq \gamma \leq 2, \\ 4, & \gamma=3,\end{cases}$

$\chi^{*}\left(v_{\gamma 2} v_{(\gamma+1) 2}\right)=\gamma+7, \quad$ for $1 \leq \gamma \leq 3$,

$\chi^{*}\left(v_{\gamma \delta} v_{(\gamma+1) \delta}\right)=\gamma+7 \delta-7, \quad$ for $1 \leq \gamma \leq 3$ and $3 \leq \delta \leq n$.

Hence, for $n \geq 2$ and $m \leq 4$, the graph $P_{m} \times P_{n}$ is a classical mean graph.

A classical mean labeling of $P_{2} \times P_{4}, P_{3} \times P_{8}$, and $P_{4} \times$ $P_{6}$ are shown in Figure 4 .

Corollary 1. Every Ladder graph $L_{n}=P_{2} \times P_{n}$ is a classical mean graph.

A classical mean labeling of $P_{2} \times P_{5}$ is shown in Figure 5 .

Theorem 4. For $m \leq 2$ and $n \geq 2$, the graph $L_{n} \circ S_{m}$ is a classical mean graph.

Proof. Case (i). $m=1$.

Construct a mapping $\chi$ from $V\left(L_{n} \circ S_{1}\right)$ to $\{1,2,3, \ldots, 5 n-1\}$ :

$$
\begin{aligned}
& \chi\left(u_{\gamma}\right)= \begin{cases}3, & \gamma=1, \\
5 \gamma-3, & 2 \leq \gamma \leq n,\end{cases} \\
& \chi\left(v_{\gamma}\right)= \begin{cases}4, & \gamma=1, \\
5 \gamma-2, & 2 \leq \gamma \leq n,\end{cases} \\
& \chi\left(u_{1}^{(\gamma)}\right)=5 \gamma-4, \quad \text { for } 1 \leq \gamma \leq n, \\
& \chi\left(x_{1}^{(\gamma)}\right)= \begin{cases}2, & \gamma=1, \\
5 \gamma-1, & 2 \leq \gamma \leq n .\end{cases}
\end{aligned}
$$

Therefore,

$$
\begin{aligned}
& \chi^{*}\left(u_{\gamma} u_{\gamma+1}\right)=5 \gamma-1, \quad \text { for } 1 \leq \gamma \leq n-1, \\
& \chi^{*}\left(v_{\gamma} v_{\gamma+1}\right)=5 \gamma, \quad \text { for } 1 \leq \gamma \leq n-1 \text {, } \\
& \chi^{*}\left(u_{\gamma} v_{\gamma}\right)= \begin{cases}3, & \gamma=1, \\
5 \gamma-3, & 2 \leq \gamma \leq n,\end{cases} \\
& \chi^{*}\left(u_{\gamma} w_{1}^{(\gamma)}\right)=5 \gamma-4, \quad \text { for } 1 \leq \gamma \leq n \text {, } \\
& \chi^{*}\left(v_{\gamma} x_{1}^{(\gamma)}\right)= \begin{cases}2, & \gamma=1, \\
5 \gamma-2, & 2 \leq \gamma \leq n .\end{cases}
\end{aligned}
$$

Case (ii). $m=2$.

Construct a mapping $\chi$ from $V\left(L_{n} \circ S_{2}\right)$ to $\{1,2,3, \ldots, 7 n-1\}$ :

$\chi\left(u_{\gamma}\right)= \begin{cases}3, & \gamma=1, \\ -2+7 \gamma, & \gamma \text { is even and } 2 \leq \gamma \leq n, \\ 7 \gamma-5, & \gamma \text { is odd and } 2 \leq \gamma \leq n,\end{cases}$

$\chi\left(v_{\gamma}\right)= \begin{cases}4, & \gamma=1, \\ 7 \gamma-4, & \gamma \text { is even and } 2 \leq \gamma \leq n, \\ 7 \gamma-1, & \gamma \text { is odd and } 2 \leq \gamma \leq n,\end{cases}$

$\chi\left(w_{1}^{(\gamma)}\right)= \begin{cases}1, & \gamma=1, \\ -3+7 \gamma, & \gamma \text { is even and } 2 \leq \gamma \leq n, \\ -6+7 \gamma, & \gamma \text { is odd and } 2 \leq \gamma \leq n,\end{cases}$

$\chi\left(w_{2}^{(\gamma)}\right)= \begin{cases}2, & \gamma=1, \\ -1+7 \gamma, & \gamma \text { is even and } 2 \leq \gamma \leq n, \\ -4+7 \gamma, & \gamma \text { is odd and } 2 \leq \gamma \leq n,\end{cases}$

$\chi\left(x_{1}^{(\gamma)}\right)= \begin{cases}2 \gamma+3, & 1 \leq \gamma \leq 2, \\ -6+7 \gamma, & \gamma \text { is even and } 2 \leq \gamma \leq n, \\ -3+7 \gamma, & \gamma \text { is odd and } 2 \leq \gamma \leq n,\end{cases}$

$\chi\left(x_{2}^{(\gamma)}\right)= \begin{cases}8, & \gamma=1, \\ -5+7 \gamma, & \gamma \text { is even and } 2 \leq \gamma \leq n, \\ -2+7 \gamma & \gamma \text { is odd and } 2 \leq \gamma \leq n .\end{cases}$

Therefore, 


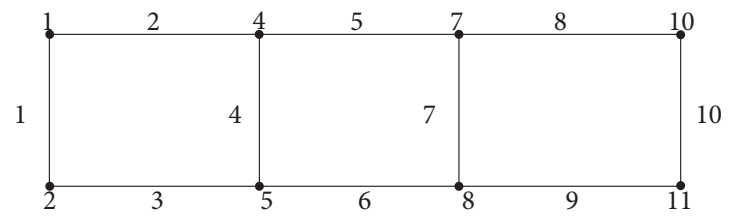

(a)

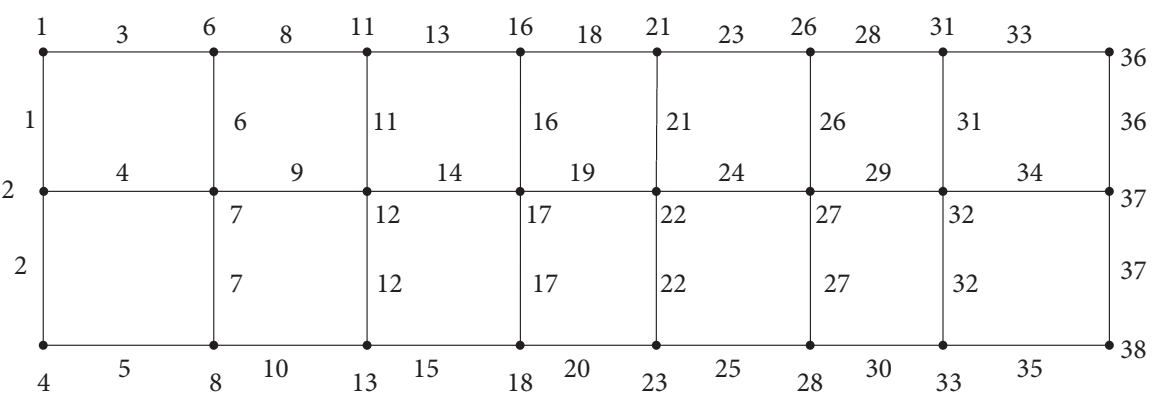

(b)

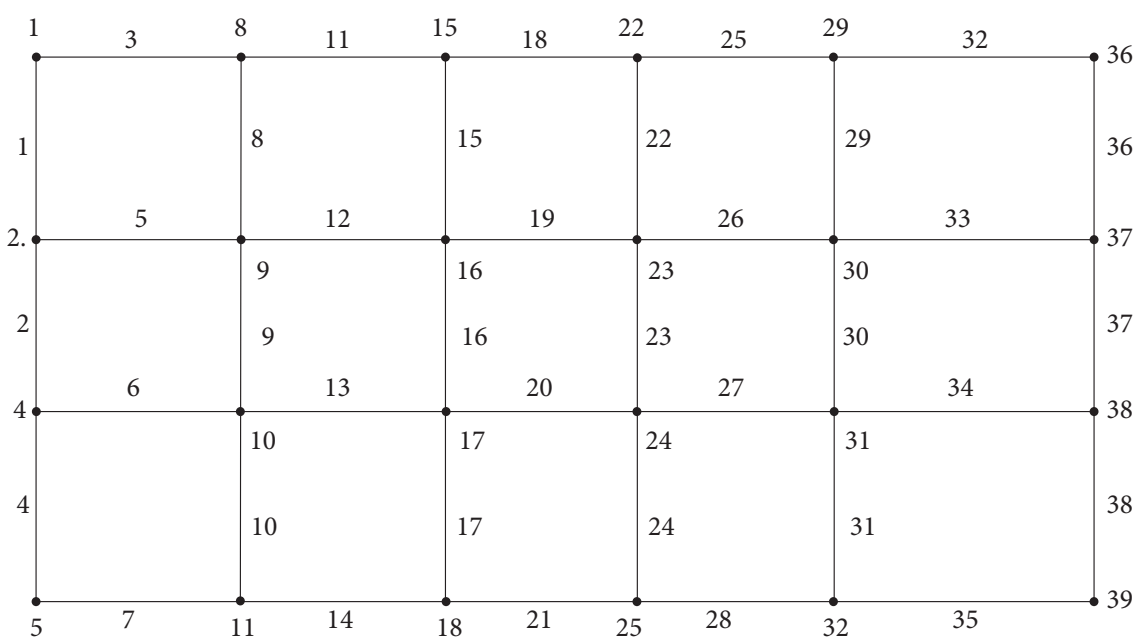

(c)

Figure 4: A classical mean labeling of $P_{2} \times P_{4}, P_{3} \times P_{8}$, and $P_{4} \times P_{6}$.

$\chi^{*}\left(u_{\gamma} u_{\gamma+1}\right)= \begin{cases}5, & \gamma=1, \\ 7 \gamma-1, & 2 \leq \gamma \leq n-1,\end{cases}$

$\chi^{*}\left(v_{\gamma} v_{\gamma+1}\right)=7 \gamma, \quad$ for $1 \leq \gamma \leq n-1$,

$\chi^{*}\left(u_{\gamma} v_{\gamma}\right)=7 \gamma-4, \quad$ for $1 \leq \gamma \leq n$,

$\chi^{*}\left(u_{\gamma} w_{1}^{(\gamma)}\right)= \begin{cases}1, & \gamma=1, \\ 7 \gamma-3, & 2 \leq \gamma \leq n \text { and } \gamma \text { is even, } \\ 7 \gamma-6, & 1 \leq \gamma \leq n \text { and } \gamma \text { isodd, }\end{cases}$

$\chi^{*}\left(u_{\gamma} w_{2}^{(\gamma)}\right)= \begin{cases}7 \gamma-2, & 1 \leq \gamma \leq n \text { and } \gamma \text { is even, } \\ 7 \gamma-5, & 1 \leq \gamma \leq n \text { and } \gamma \text { isodd, }\end{cases}$

$\chi^{*}\left(v_{\gamma} x_{1}^{(\gamma)}\right)= \begin{cases}7 \gamma-6, & 1 \leq \gamma \leq n \text { and } \gamma \text { is even, } \\ 7 \gamma-3, & 4 \leq \gamma \leq n \text { and } \gamma \text { isodd, }\end{cases}$

$\chi^{*}\left(v_{\gamma} x_{2}^{(\gamma)}\right)= \begin{cases}7 \gamma-5, & 2 \leq \gamma \leq n \text { and } \gamma \text { is even } \\ 7 \gamma-2, & 1 \leq \gamma \leq n \text { and } \gamma \text { isodd. }\end{cases}$

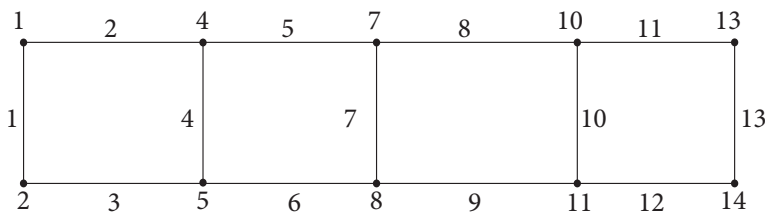

Figure 5: A classical mean labeling of $P_{2} \times P_{5}$.

Hence, for $m \leq 2$ and $n \geq 2$, the graph $L_{n} \circ S_{m}$ is a classical mean graph.

A classical mean labeling of $L_{5} \circ S_{1}$ is shown in Figure 6 .

A classical mean labeling of $L_{5} \circ S_{2}$ is shown in Figure 7 .

Theorem 5. The triangular ladder graph $T L_{n}$ is a classical mean graph, for $n \geq 2$.

Proof. Construct a mapping $\chi$ from $V\left(\mathrm{TL}_{n}\right)$ to $\{1,2,3, \ldots, 4 n-2\}$ : 


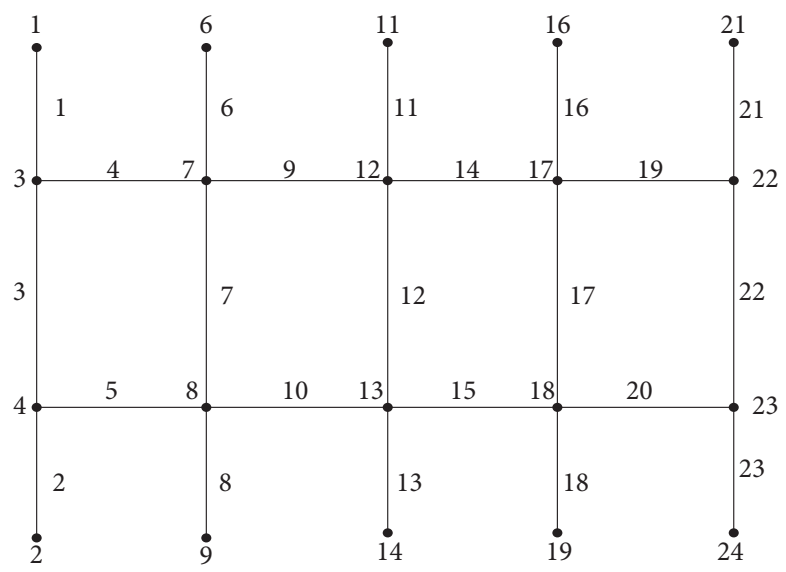

Figure 6: A classical mean labeling of $L_{5} \circ S_{1}$.

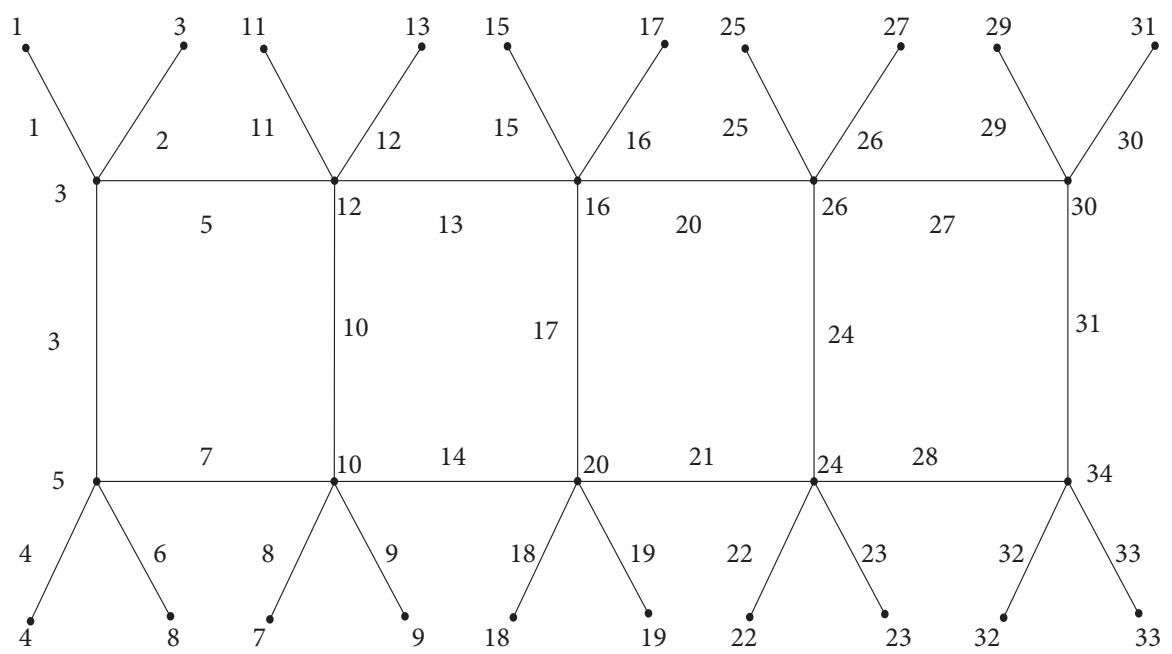

Figure 7: A classical mean labeling of $L_{5} \circ S_{2}$.

$$
\begin{aligned}
& \chi\left(u_{\gamma}\right)=4 \gamma-3, \quad \text { for } 1 \leq \gamma \leq n, \\
& \chi\left(v_{\gamma}\right)= \begin{cases}4 \gamma-1, & 1 \leq \gamma \leq n-1, \\
4 n-2, & \gamma=n\end{cases}
\end{aligned}
$$

Therefore,

$$
\begin{aligned}
\chi^{*}\left(u_{\gamma} u_{\gamma+1}\right) & =4 \gamma-2, \quad \text { for } 1 \leq \gamma \leq n-1, \\
\chi^{*}\left(u_{\gamma} v_{\gamma}\right) & =4 \gamma-3, \quad \text { for } 1 \leq \gamma \leq n, \\
\chi^{*}\left(v_{\gamma} v_{\gamma+1}\right) & =4 \gamma, \quad \text { for } 1 \leq \gamma \leq n-1, \\
\chi^{*}\left(u_{\gamma} v_{\gamma+1}\right) & =-1+4 \gamma, \quad \text { for } 1 \leq \gamma \leq n-1 .
\end{aligned}
$$

Hence, the triangular ladder graph $\mathrm{TL}_{n}$ is a classical mean graph, for $n \geq 2$.

A classical mean labeling of $\mathrm{TL}_{8}$ is shown in Figure 8.
Theorem 6. For $m \leq 2$ and $n \geq 2$, the graph $T L_{n} \circ S_{m}$ is a classical mean graph.

Proof. Case (i). $m=1$.

Construct a mapping $\chi$ from $V\left(L_{n} \circ S_{1}\right)$ to $\{1,2,3, \ldots, 6 n-2\}$ :

$$
\begin{aligned}
& \chi\left(u_{\gamma}\right)= \begin{cases}5 \gamma-3, & 1 \leq \gamma \leq 2, \\
6 \gamma-4, & 3 \leq \gamma \leq n,\end{cases} \\
& \chi\left(v_{\gamma}\right)=6 \gamma-2, \quad \text { for } 1 \leq \gamma \leq n \text {, } \\
& \chi\left(w_{1}^{(\gamma)}\right)= \begin{cases}7 \gamma-6, & 1 \leq \gamma \leq 2, \\
6 \gamma-5, & 3 \leq \gamma \leq n,\end{cases} \\
& \chi\left(x_{1}^{(\gamma)}\right)= \begin{cases}3, & \gamma=1, \\
6 \gamma-3, & 2 \leq \gamma \leq n .\end{cases}
\end{aligned}
$$

Therefore, 


$$
\begin{aligned}
& \chi^{*}\left(u_{\gamma} u_{\gamma+1}\right)=-2+6 \gamma, \quad \text { for } 1 \leq \gamma \leq n-1, \\
& \chi^{*}\left(v_{\gamma} v_{\gamma+1}\right)=6 \gamma, \quad \text { for } 1 \leq \gamma \leq n-1 \text {, } \\
& \chi^{*}\left(u_{\gamma} v_{\gamma+1}\right)=6 \gamma-1, \quad \text { for } 1 \leq \gamma \leq n-1 \text {, } \\
& \chi^{*}\left(u_{\gamma} v_{\gamma}\right)= \begin{cases}2, & \gamma=1, \\
6 \gamma-4, & 2 \leq \gamma \leq n,\end{cases} \\
& \chi^{*}\left(u_{\gamma} w_{1}^{(\gamma)}\right)=6 \gamma-5, \quad \text { for } 1 \leq \gamma \leq n, \\
& \chi^{*}\left(v_{\gamma} x_{1}^{(\gamma)}\right)= \begin{cases}3, & \gamma=1, \\
6 \gamma-3, & 2 \leq \gamma \leq n .\end{cases}
\end{aligned}
$$

Case (ii). $m=2$.

Construct a mapping $\chi$ from $V\left(L_{n} \circ S_{2}\right)$ to $\{1,2,3, \ldots, 8 n-2\}$ :

$$
\begin{aligned}
\chi\left(u_{\gamma}\right) & = \begin{cases}2, & \gamma=1, \\
8 \gamma-3, & 2 \leq \gamma \leq n,\end{cases} \\
\chi\left(v_{\gamma}\right) & = \begin{cases}6, & \gamma=1, \\
8 \gamma-5, & 2 \leq \gamma \leq n,\end{cases} \\
\chi\left(w_{1}^{(\gamma)}\right) & = \begin{cases}1, & \gamma=1, \\
8 \gamma-4, & 2 \leq \gamma \leq n,\end{cases} \\
\chi\left(w_{2}^{(\gamma)}\right) & = \begin{cases}3, & \gamma=1, \\
8 \gamma-2, & 2 \leq \gamma \leq n,\end{cases} \\
\chi\left(x_{1}^{(\gamma)}\right) & = \begin{cases}4, & \gamma=1 \\
8 \gamma-7, & 2 \leq \gamma \leq n,\end{cases} \\
\chi\left(x_{2}^{(\gamma)}\right) & = \begin{cases}9, & \gamma=1, \\
8 \gamma-6, & 2 \leq \gamma \leq n .\end{cases}
\end{aligned}
$$

Therefore,

$$
\begin{aligned}
& \chi^{*}\left(u_{\gamma} u_{\gamma+1}\right)= \begin{cases}6, & \gamma=1, \\
8 \gamma, & 2 \leq \gamma \leq n-1,\end{cases} \\
& \chi^{*}\left(v_{\gamma} v_{\gamma+1}\right)= \begin{cases}8, & \gamma=1, \\
8 \gamma-2, & 2 \leq \gamma \leq n-1,\end{cases} \\
& \chi^{*}\left(u_{\gamma} v_{\gamma}\right)=8 \gamma-5, \quad \text { for } 1 \leq \gamma \leq n, \\
& \chi^{*}\left(u_{\gamma} v_{\gamma+1}\right)= \begin{cases}5, & \gamma=1, \\
8 \gamma-1, & 2 \leq \gamma \leq n-1,\end{cases} \\
& \chi^{*}\left(u_{\gamma} w_{1}^{(\gamma)}\right)= \begin{cases}1, & \gamma=1, \\
8 \gamma-4, & 2 \leq \gamma \leq n,\end{cases} \\
& \chi^{*}\left(u_{\gamma} w_{2}^{(\gamma)}\right)= \begin{cases}2, & \gamma=1, \\
8 \gamma-3, & 2 \leq \gamma \leq n,\end{cases} \\
& \chi^{*}\left(v_{\gamma} x_{1}^{(\gamma)}\right)= \begin{cases}4, & \gamma=1, \\
8 \gamma-5, & 2 \leq \gamma \leq n,\end{cases} \\
& \chi^{*}\left(v_{\gamma} x_{2}^{(\gamma)}\right)= \begin{cases}7, & \gamma=1, \\
8 \gamma-6, & 2 \leq \gamma \leq n .\end{cases}
\end{aligned}
$$

Hence, for $m \leq 2$ and $n \geq 2$, the graph $\mathrm{TL}_{n} \circ S_{m}$ is a classical mean graph.
A classical mean labeling of $\mathrm{TL}_{5} \circ S_{1}$ is shown in Figure 9 .

A classical mean labeling of $\mathrm{TL}_{4} \circ S_{2}$ is shown in Figure 10.

Theorem 7. For $n \geq 2$, the slanting ladder graph $S L_{n}$ is a classical mean graph.

Proof. Construct a mapping $\chi$ from $V\left(\mathrm{SL}_{n}\right)$ to $\{1,2,3, \ldots, 3 n-2\}$ :

$$
\begin{aligned}
& \chi\left(u_{1}\right)=1, \\
& \chi\left(u_{\gamma}\right)=-4+3 \gamma, \quad \text { for } 2 \leq \gamma \leq n, \\
& \chi\left(v_{\gamma}\right)=3 \gamma, \quad \text { for } 1 \leq \gamma \leq-1+n, \\
& \chi\left(v_{n}\right)=-2+3 n .
\end{aligned}
$$

Therefore,

$$
\begin{aligned}
& \chi^{*}\left(u_{\gamma} u_{\gamma+1}\right)= \begin{cases}1, & \gamma=1, \\
3 \gamma-3, & 2 \leq \gamma \leq n-1,\end{cases} \\
& \chi^{*}\left(v_{\gamma} v_{\gamma+1}\right)=3 \gamma+1, \quad \text { for } 1 \leq \gamma \leq n-2, \\
& \chi^{*}\left(v_{n-1} v_{n}\right)=3 n-3, \\
& \chi^{*}\left(v_{\gamma} u_{\gamma+1}\right)=3 \gamma-1, \quad \text { for } 1 \leq \gamma \leq n-1 .
\end{aligned}
$$

Hence, for $n \geq 2$, the slanting ladder graph $\mathrm{SL}_{n}$ is a classical mean graph.

A classical mean labeling of $\mathrm{SL}_{8}$ is shown in Figure 11.

Theorem 8. For $m \leq 2$ and $n \geq 2$, the graph $S L_{n} \circ S_{m}$ is a classical mean graph.

Proof. Let $\quad E\left(\mathrm{SL}_{n} \circ S_{m}\right)=\left\{u_{\gamma} u_{\gamma+1}, \quad v_{\gamma} v_{\gamma+1}: 1 \leq \gamma \leq\right.$ $n-1\} \cup\left\{u_{\gamma} v_{\gamma-1}: 2 \leq \gamma \leq n\right\} \cup\left\{u_{\gamma} u_{\delta}^{(\gamma)}: 1 \leq \gamma \leq n, 1 \leq \delta \leq m\right\} \cup$ $\left\{v_{\gamma} v_{\delta}^{(\gamma)}: 1 \leq \gamma \leq n, 1 \leq \delta \leq m\right\}$.

Case (i). $n \geq 3$ and $m=1$.

Construct a mapping $\chi$ from $V\left(\mathrm{SL}_{n} \circ S_{1}\right)$ to $\{1,2,3, \ldots, 5 n-2\}$ :

$$
\begin{aligned}
\chi\left(u_{\gamma}\right) & = \begin{cases}1+\gamma, & 1 \leq \gamma \leq 2, \\
-6+5 \gamma, & 3 \leq \gamma \leq n,\end{cases} \\
\chi\left(v_{\gamma}\right) & = \begin{cases}6, & \gamma=1, \\
5 \gamma, & 2 \leq \gamma \leq n-1, \\
-2+5 n, & \gamma=n,\end{cases} \\
\chi\left(w_{1}^{(\gamma)}\right) & = \begin{cases}-2+3 \gamma, & 1 \leq \gamma \leq 2, \\
-7+5 \gamma, & 3 \leq \gamma \leq n,\end{cases} \\
\chi\left(x_{1}^{(\gamma)}\right) & = \begin{cases}7, & \gamma=1, \\
1+5 \gamma, & 2 \leq \gamma \leq n-1, \\
-3+5 n, & \gamma=n .\end{cases}
\end{aligned}
$$

Therefore, 


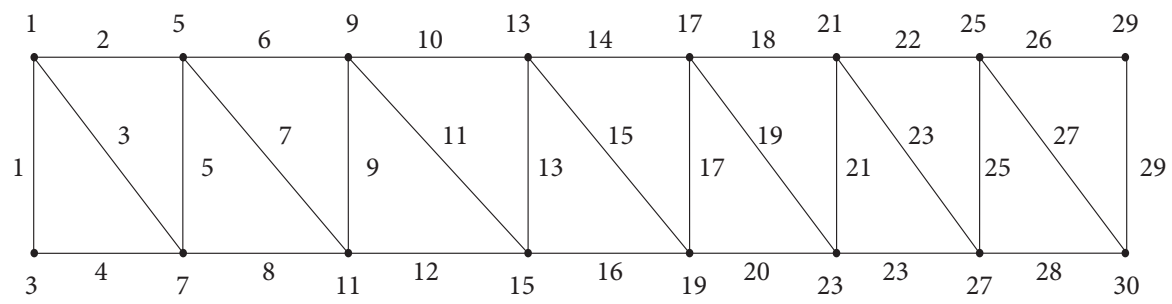

Figure 8: A classical mean labeling of $\mathrm{TL}_{8}$.

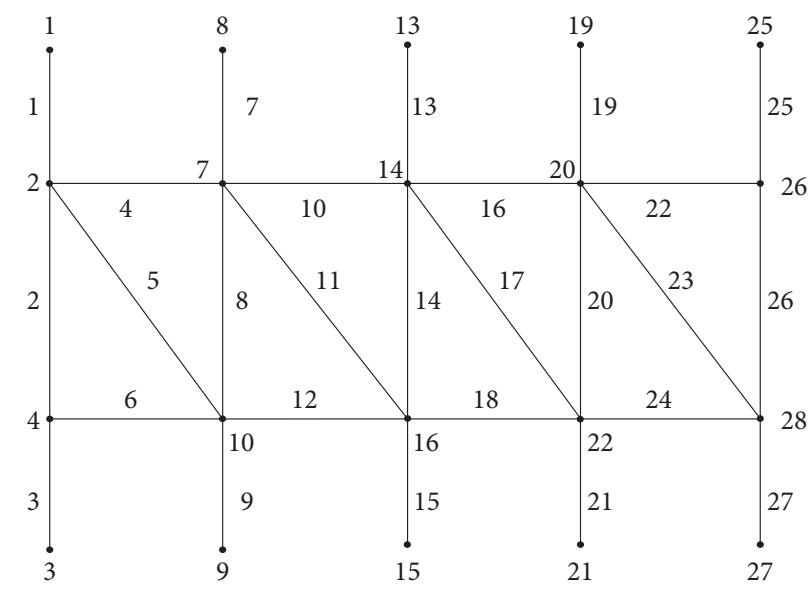

Figure 9: A classical mean labeling of $\mathrm{TL}_{5} \circ S_{1}$.

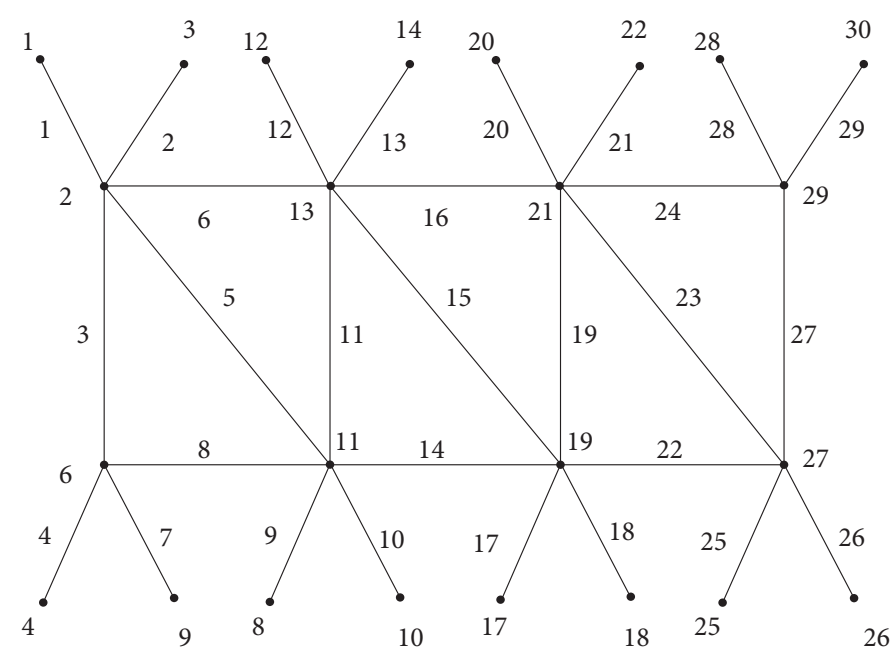

Figure 10: A classical mean labeling of $\mathrm{TL}_{4} \circ S_{2}$.

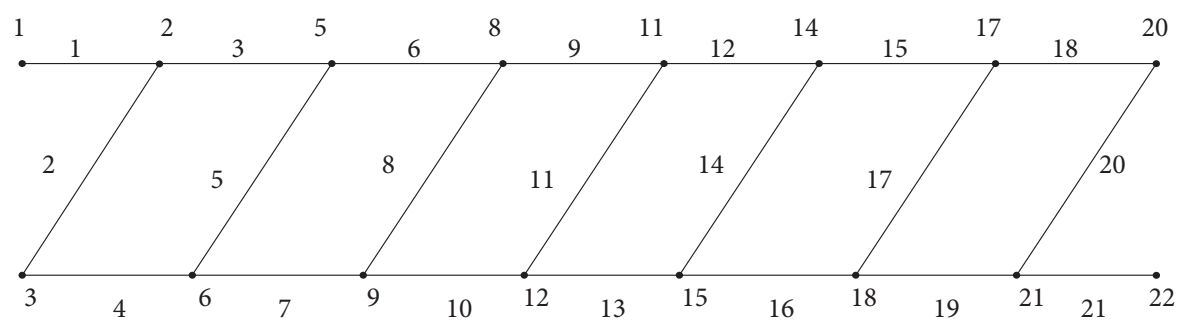

Figure 11: A classical mean labeling of $\mathrm{SL}_{8}$. 


$$
\begin{aligned}
& \chi^{*}\left(u_{\gamma} u_{\gamma+1}\right)= \begin{cases}-1+3 \gamma, & 1 \leq \gamma \leq 2, \\
-4+5 \gamma, & 3 \leq \gamma \leq n-1,\end{cases} \\
& \chi^{*}\left(v_{\gamma} v_{\gamma+1}\right)= \begin{cases}2+5 \gamma, & 1 \leq \gamma \leq n-2, \\
-4+5 n, & \gamma=n-1,\end{cases} \\
& \chi^{*}\left(v_{\gamma} u_{\gamma+1}\right)=-1+5 \gamma, \quad \text { for } 1 \leq \gamma \leq n-1 \text {, } \\
& \chi^{*}\left(u_{\gamma} w_{1}^{(\gamma)}\right)= \begin{cases}1, & \gamma=1, \\
-7+5 \gamma, & 2 \leq \gamma \leq n,\end{cases} \\
& \chi^{*}\left(v_{\gamma} x_{1}^{(\gamma)}\right)= \begin{cases}6, & \gamma=1, \\
5 \gamma, & 2 \leq \gamma \leq n-1, \\
-3+5 n, & \gamma=n .\end{cases}
\end{aligned}
$$

Case (ii). $n \geq 3$ and $m=2$.

Construct a mapping $\chi$ from $V\left(\mathrm{SL}_{n} \circ S_{2}\right)$ to $\{1,2,3, \ldots, 7 n-2\}$

$$
\begin{aligned}
& \chi\left(u_{\gamma}\right)= \begin{cases}2+\gamma, & 1 \leq \gamma \leq 2, \\
-9+7 \gamma, & \gamma \text { is odd and } 3 \leq \gamma \leq n-1, \\
-6+7 \gamma, & \gamma \text { is even and } 3 \leq \gamma \leq n-1, \\
-10+7 n, & n \text { is even and } \gamma=n, \\
-9+7 n, & n \text { is odd and } \gamma=n,\end{cases} \\
& \chi\left(v_{\gamma}\right)= \begin{cases}8, & \gamma=1, \\
-1+7 \gamma, & \gamma \text { is odd and } 2 \leq \gamma \leq n-3 \\
2+7 \gamma, & \gamma \text { is even and } 2 \leq \gamma \leq n-3 \\
-13+7 n, & n \text { is even and } \gamma=n-2, \\
-15+7 n, & n \text { is odd and } \gamma=n-2, \\
7 n-5, & \gamma=n-1, \\
7 n-3, & \gamma=n,\end{cases} \\
& \chi\left(w_{1}^{(\gamma)}\right)= \begin{cases}1, & \gamma=1 \\
-5+5 \gamma, & 2 \leq \gamma \leq 3, \\
-7+7 \gamma, & \gamma \text { is even and } 4 \leq \gamma \leq n-1, \\
-10+7 \gamma, & \gamma \text { is odd and } 4 \leq \gamma \leq n-1, \\
-11+7 n, & n \text { is even and } \gamma=n, \\
-10+7 n, & n \text { is odd and } \gamma=n,\end{cases} \\
& \chi\left(w_{2}^{(\gamma)}\right)= \begin{cases}-5+7 \gamma, & 1 \leq \gamma \leq 2, \\
-5+7 \gamma, & \gamma \text { is even and } 3 \leq \gamma \leq n-1, \\
-8+7 \gamma, & \gamma \text { is odd and } 3 \leq \gamma \leq n-1, \\
-7+7 n, & n \text { is even and } \gamma=n, \\
-8+7 n, & n \text { is odd and } \gamma=n,\end{cases}
\end{aligned}
$$

$$
\begin{aligned}
& \chi\left(x_{1}^{(\gamma)}\right)= \begin{cases}9, & \gamma=1, \\
7 \gamma, & 2 \leq \gamma \leq n-3 \text { and } \gamma \text { iseven, } \\
-3+7 \gamma, & 2 \leq \gamma \leq n-3 \text { and } \gamma \text { isodd, } \\
-12+7 n, & n \text { is even and } \gamma=n-2, \\
-17+7 n, & n \text { is odd and } \gamma=n-2, \\
-8+7 n, & n \text { is even and } \gamma=n-1, \\
-7+7 n, & n \text { is odd and } \gamma=n-1, \\
-4+7 n, & \gamma=n,\end{cases} \\
& \chi\left(x_{2}^{(\gamma)}\right)= \begin{cases}11, & \gamma=1, \\
7 \gamma+1, & \gamma \text { is even and } 2 \leq \gamma \leq n-3, \\
-2+7 \gamma, & \gamma \text { is odd and } 2 \leq \gamma \leq n-3, \\
-9+7 n, & n \text { is even and } \gamma=n-2, \\
-16+7 n, & n \text { is odd and } \gamma=n-2, \\
-6+7 n, & \gamma=n-1, \\
-2+7 n, & \gamma=n .\end{cases}
\end{aligned}
$$

Therefore,

$$
\chi^{*}\left(u_{\gamma} u_{\gamma+1}\right)= \begin{cases}-1+4 \gamma, & 1 \leq \gamma \leq 2, \\ -5+7 \gamma, & 3 \leq \gamma \leq n-2, \\ -14+7 n, & n \text { is even and } \gamma=n-1, \\ -12+7 n, & n \text { is odd and } \gamma=n-1,\end{cases}
$$

$$
\chi^{*}\left(v_{\gamma} v_{\gamma+1}\right)= \begin{cases}11, & \gamma=1, \\ 3+7 \gamma, & 2 \leq \gamma \leq n-3, \\ -10+7 n, & n \text { is even and } \gamma=n-2, \\ -11+7 n, & n \text { is odd and } \gamma=n-2, \\ -5+7 n, & \gamma=n-1,\end{cases}
$$$$
\chi^{*}\left(v_{\gamma} u_{\gamma+1}\right)= \begin{cases}5, & \gamma=1, \\ -1+7 \gamma, & 2 \leq \gamma \leq n-1,\end{cases}
$$$$
\chi^{*}\left(u_{\gamma} w_{1}^{(\gamma)}\right)= \begin{cases}1, & \gamma=1, \\ -8+6 \gamma, & 2 \leq \gamma \leq 3, \\ -7+7 \gamma, & \gamma \text { is even and } 4 \leq \gamma \leq n-1, \\ -10+7 \gamma, & \gamma \text { is odd and } 4 \leq \gamma \leq n-1, \\ -11+7 n, & n \text { is even and } \gamma=n, \\ -10+7 n, & n \text { is odd and } \gamma=n,\end{cases}
$$$$
\chi^{*}\left(u_{\gamma} w_{2}^{(\gamma)}\right)= \begin{cases}-2+4 \gamma, & 1 \leq \gamma \leq 2, \\ -6+7 \gamma, & \gamma \text { is even and } 3 \leq \gamma \leq n-1, \\ -9+7 \gamma, & \gamma \text { is odd and } 3 \leq \gamma \leq n-1, \\ -9+7 n, & \gamma=n,\end{cases}
$$ 


$$
\begin{aligned}
& \chi^{*}\left(v_{\gamma} x_{1}^{(\gamma)}\right)= \begin{cases}8, & \gamma=1, \\
7 \gamma, & \gamma \text { is even and } 2 \leq \gamma \leq n-3, \\
-3+7 \gamma, & \gamma \text { is odd and } 2 \leq \gamma \leq n-3, \\
-13+7 n, & n \text { is even and } \gamma=n-2, \\
-17+7 n, & n \text { is odd and } \gamma=n-2, \\
-7+7 n, & \gamma=n-1, \\
-4+7 n, & \gamma=n,\end{cases} \\
& \chi^{*}\left(v_{\gamma} x_{2}^{(\gamma)}\right)= \begin{cases}9, & \gamma=1, \\
1+7 \gamma, & \gamma \text { is even and } 2 \leq \gamma \leq n-3, \\
-2+7 \gamma, & \gamma \text { is odd and } 2 \leq \gamma \leq n-3, \\
-12+7 n, & n \text { is even and } \gamma=n-2, \\
-16+7 n, & n \text { is odd and } \gamma=n-2, \\
-6+7 \gamma, & \gamma=n-1, \\
-3+7 n, & \gamma=n .\end{cases}
\end{aligned}
$$

Case (iii). $n=2$ and $m=1,2$.

A classical mean labeling of $\mathrm{SL}_{2} \circ S_{1}$ and $\mathrm{SL}_{2} \circ S_{2}$ are shown in Figure 12.

A classical mean labeling of $\mathrm{SL}_{6} \circ S_{1}$ is shown in Figure 13.

A classical mean labeling of $\mathrm{SL}_{5} \circ S_{2}$ is shown in Figure 14.

Theorem 9. For $n \geq 2$, the graph $D_{n}^{*}$ is a classical mean graph.

Proof. Take $V\left(D_{n}^{*}\right)=\left\{a_{\gamma, \delta}: 1 \leq \gamma \leq n, \delta=1,2,3,4\right\}$ and $E\left(D_{n}^{*}\right)=\left\{a_{\gamma, 1} a_{\gamma+1,1}, a_{\gamma, 3} a_{\gamma+1,3}: 1 \leq \gamma \leq n-1\right\} \cup\left\{a_{\gamma, 1} a_{\gamma, 2}, a_{\gamma, 2}\right.$ $\left.a_{\gamma, 3}, a_{\gamma, 3} a_{\gamma, 4}, a_{\gamma, 4} a_{\gamma, 1}: 1 \leq \gamma \leq n\right\}$.

Construct a mapping $\chi$ from $V\left(D_{n}^{*}\right)$ to $\{1,2,3, \ldots, 6 n-1\}$ :

$$
\begin{array}{ll}
\chi\left(a_{\gamma, 1}\right)=-2+6 \gamma, & \text { for } 1 \leq \gamma \leq n, \\
\chi\left(a_{\gamma, 2}\right)=-5+6 \gamma, & \text { for } 1 \leq \gamma \leq n, \\
\chi\left(a_{\gamma, 3}\right)=-3+6 \gamma, & \text { for } 1 \leq \gamma \leq n, \\
\chi\left(a_{\gamma, 4}\right)=-1+6 \gamma, & \text { for } 1 \leq \gamma \leq n .
\end{array}
$$

Therefore,

$$
\begin{aligned}
& \chi^{*}\left(a_{\gamma, 1} a_{\gamma+1,1}\right)=6 \gamma, \quad \text { for } 1 \leq \gamma \leq n-1, \\
& \chi^{*}\left(a_{\gamma, 3} a_{\gamma+1,3}\right)=-1+6 \gamma, \text { for } 1 \leq \gamma \leq n-1, \\
& \chi^{*}\left(a_{\gamma, 1} a_{\gamma, 2}\right)=-4+6 \gamma, \text { for } 1 \leq \gamma \leq n, \\
& \chi^{*}\left(a_{\gamma, 2} a_{\gamma, 3}\right)=-5+6 \gamma, \text { for } 1 \leq \gamma \leq n, \\
& \chi^{*}\left(a_{\gamma, 3} a_{\gamma, 4}\right)=-3+6 \gamma, \text { for } 1 \leq \gamma \leq n, \\
& \chi^{*}\left(a_{\gamma, 4} a_{\gamma, 1}\right)=-2+6 \gamma, \text { for } 1 \leq \gamma \leq n .
\end{aligned}
$$

Hence, for $n \geq 2$, the graph $D_{n}^{*}$ is a classical mean graph.
A classical mean labeling of $D_{4}^{*}$ is shown in Figure 15.

Theorem 10. For $n \geq 1$, the diamond ladder graph $D l_{n}$ is a classical mean graph.

Proof. $V\left(D l_{n}\right)=\left\{x_{\gamma}, y_{\gamma}: 1 \leq \gamma \leq n\right\} \cup\left\{z_{\gamma}: 1 \leq \gamma \leq 2 n\right\}$ and $E\left(D l_{n}\right)=\left\{x_{\gamma} y_{\gamma}: 1 \leq \gamma \leq n\right\} \cup\left\{x_{\gamma} x_{\gamma+1}, y_{\gamma} y_{\gamma+1}: 1 \leq \gamma \leq n-1\right\}$ $\cup\left\{x_{\gamma} z_{2 \gamma-1}, x_{\gamma} z_{2 \gamma}, y_{\gamma} z_{2 \gamma-1}, y_{\gamma} z_{2 \gamma}: 1 \leq \gamma \leq n\right\} \cup\left\{z_{2 \gamma} z_{2 \gamma+1}: 1 \leq\right.$ $\gamma \leq n-1\}$. Thus, $\left|V\left(D l_{n}\right)\right|=4 n$ and $\left|E\left(D l_{n}\right)\right|=8 n-3$.

Construct a mapping $\chi$ from $V\left(D l_{n}\right)$ to $\{1,2,3, \ldots, 8 n-2\}$ :

$\chi\left(x_{\gamma}\right)=-5+8 \gamma, \quad$ for $1 \leq \gamma \leq n$,

$\chi\left(y_{\gamma}\right)=-3+8 \gamma, \quad$ for $1 \leq \gamma \leq n$,

$\chi\left(z_{\gamma}\right)= \begin{cases}1, & \gamma=1, \\ 4 \gamma-\left(\frac{(-1)^{\gamma+1}+1}{2}\right)-2, & \gamma \text { is even and } 2 \leq \gamma \leq 2 n, \\ 4 \gamma-\left(\frac{(-1)^{\gamma+1}+1}{2}\right)-2, & \gamma \text { is odd and } 3 \leq \gamma \leq 2 n .\end{cases}$

Therefore,

$$
\begin{aligned}
\chi^{*}\left(x_{\gamma} x_{\gamma+1}\right) & =-2+8 \gamma, \quad \text { for } 1 \leq \gamma \leq n-1, \\
\chi^{*}\left(y_{\gamma} y_{\gamma+1}\right) & =8 \gamma, \quad \text { for } 1 \leq \gamma \leq n-1, \\
\chi^{*}\left(x_{\gamma} y_{\gamma}\right) & =-5+8 \gamma, \quad \text { for } 1 \leq \gamma \leq n, \\
\chi^{*}\left(z_{2 \gamma} z_{2 \gamma+1}\right) & =-1+8 \gamma, \quad \text { for } 1 \leq \gamma \leq n-1, \\
\chi^{*}\left(x_{\gamma} z_{2 \gamma-1}\right) & =-7+8 \gamma, \quad \text { for } 1 \leq \gamma \leq n, \\
\chi^{*}\left(x_{\gamma} z_{2 \gamma}\right) & =-4+8 \gamma, \quad \text { for } 1 \leq \gamma \leq n, \\
\chi^{*}\left(y_{\gamma} z_{2 \gamma-1}\right) & =-6+8 \gamma, \quad \text { for } 1 \leq \gamma \leq n, \\
\chi^{*}\left(y_{\gamma} z_{2 \gamma}\right) & =-3+8 \gamma, \quad \text { for } 1 \leq \gamma \leq n .
\end{aligned}
$$

Hence, for $n \geq 1$, the diamond ladder graph $D l_{n}$ is a classical mean graph.

A classical mean labeling of $\mathrm{Dl}_{4}$ is shown in Figure 16.

Theorem 11. For $n \geq 4$, the latitude ladder graph $L L_{n}$ is a classical mean graph.

Proof. Here, $V\left(\mathrm{LL}_{n}\right)=\left\{u_{\gamma}: 1 \leq \gamma \leq n\right\}$ and $E\left(L L_{n}\right)=\left\{u_{\gamma}\right.$ $\left.u_{\gamma+1}: 1 \leq \gamma \leq n-1\right\} \cup\left\{u_{n} u_{1}\right\} \cup\left\{u_{\gamma} u_{n+2-\gamma}: 2 \leq \gamma \leq(n / 2)\right\}$.

Construct a mapping $\chi$ from $V\left(\mathrm{LL}_{n}\right)$ to $\{1,2,3, \ldots,(3 n / 2)\}$ 


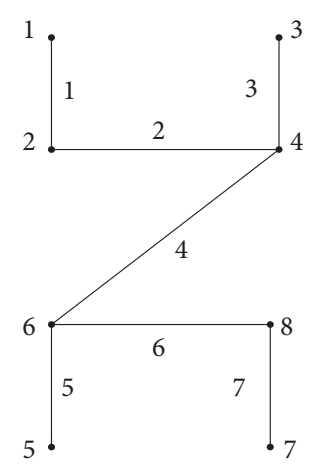

(a)

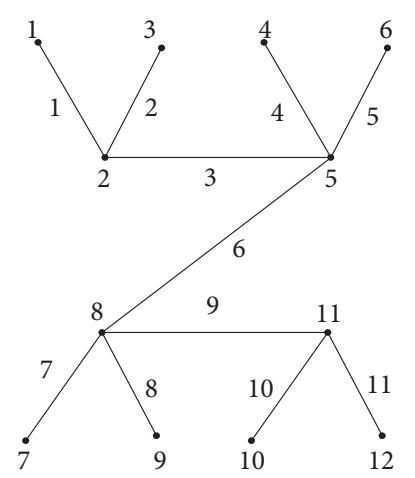

(b)

FIgURE 12: A classical mean labeling of $\mathrm{SL}_{2} \circ S_{1}$ and $\mathrm{SL}_{2} \circ S_{2}$.

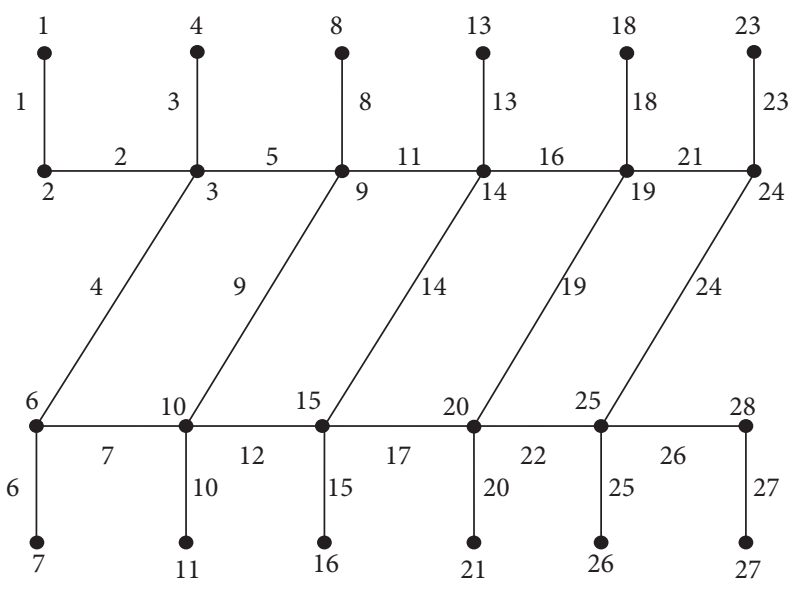

Figure 13: A classical mean labeling of $\mathrm{SL}_{6} \circ S_{1}$.

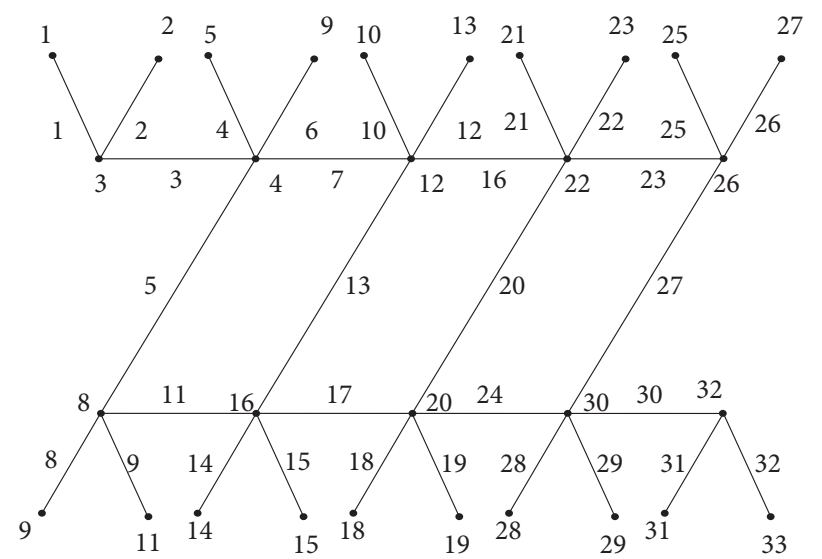

FIgURe 14: A classical mean labeling of $\mathrm{SL}_{5} \circ S_{2}$.

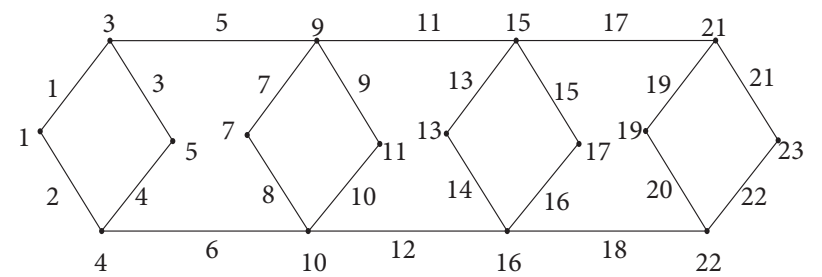

FIgURE 15: A classical mean labeling of $D_{4}^{*}$. 


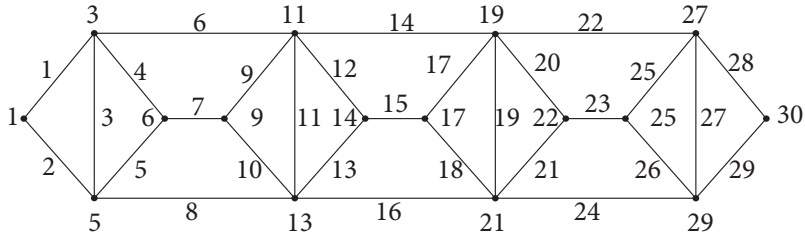

Figure 16: A classical mean labeling of $\mathrm{Dl}_{4}$.

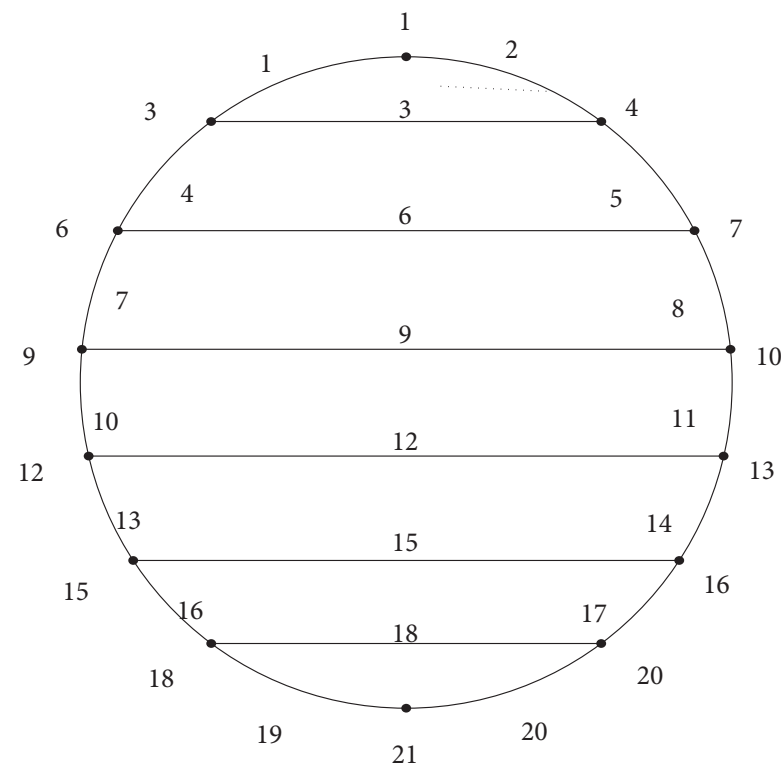

FIgURe 17: A classical mean labeling of latitude graph $\mathrm{LL}_{14}$.

$$
\chi\left(u_{\gamma}\right)= \begin{cases}3 \gamma-2, & 1 \leq \gamma \leq \frac{n}{2}-1, \\ 3 \gamma-1, & \gamma=\frac{n}{2}, \\ \frac{3 n}{2}, & \gamma=1+\frac{n}{2} \\ 3 \gamma+3 n-+3, & 2+\frac{n}{2} \leq \gamma \leq n\end{cases}
$$

Therefore,

$$
\begin{aligned}
\chi^{*}\left(u_{\gamma} u_{\gamma+1}\right) & = \begin{cases}3 \gamma-1, & 1 \leq \gamma \leq \frac{n}{2}, \\
3 n-3 \gamma+1, & \frac{n}{2}+1 \leq \gamma \leq n,\end{cases} \\
\chi^{*}\left(u_{\gamma} u_{n+2-\gamma}\right) & =3 \gamma-3, \quad \text { for } 2 \leq \gamma \leq \frac{n}{2} .
\end{aligned}
$$

Hence, for $n \geq 4$, the latitude ladder graph $\mathrm{LL}_{n}$ is a classical mean graph.

A classical mean labeling of $\mathrm{LL}_{14}$ is shown in Figure 17.

\section{Conclusion}

The Cartesian product is one among graph operations. Based on this operation, the classical mean labeling of various graphs such as the one-sided step graph $\mathrm{ST}_{n}$, double-sided step graph $2 \mathrm{ST}_{2 n}$, graph $P_{m} \times P_{n}$, ladder graph $L_{n}$, graph $L_{n} \circ S_{m}$, triangular ladder graph $\mathrm{TL}_{n}$, graph $\mathrm{TL}_{n} \circ S_{m}$, slanting ladder graph $\mathrm{SL}_{n}$, graph $\mathrm{SL}_{n} \circ S_{m}$, graph $D_{n}^{*}$, diamond ladder graph $D l_{n}$, and latitude ladder graph $\mathrm{LL}_{n}$ are established. It would be very interesting to analyze that the classical meanness of various ladder-related graphs. Investigating classical mean labeling of other classes of graphs is still open and this is for future work. One can also explore the exclusive applications of classical mean labeling in real-life problems.

\section{Data Availability}

No data were used to support the study.

\section{Conflicts of Interest}

The authors declare that they have no conflicts of interest.

\section{References}

[1] F. Harary, Graph Theory, Narosa Publishing House Reading, New Delhi, India, 1988.

[2] B. D. Acharya, S. Arumugam, and A. Rosa, Labeling of Discrete Structures and Applications, Narosa Publishing House Reading, New Delhi, India, 2008.

[3] J. Gross and J. Yellen, Graph Theory and its Applications, CRC Press, London, UK, 1999.

[4] G. Chartrand, L. Lesniak, and P. Zhang, Graphs and Digraphs, Taylor \& Francis Group, Boca Raton, FL, USA, 6th edition, 2016.

[5] F. Buckley and F. Harary, Distance in Graphs, AddisonWesley, Boston, MA, USA, 1990.

[6] S. W. Golomb, How to Number a Graph in Graph Theory and Computing, Academic Press, Cambridge, MA, USA, 1972.

[7] J. A. Gallian, "A dynamic survey of graph labeling," The Electronic Journal of Combinatorics, vol. 19, 2019.

[8] A. Rosa, "On certain valuations of the vertices of a graph," Journal of Graph Theory, vol. 5, 1967.

[9] S. Somasundaram and R. Ponraj, "Mean labelings of graphs," National Academy Science Letters, vol. 26, pp. 210-213, 2003.

[10] S. Arockiaraj, A. Durai Baskar, and A. Rajesh Kannan, " $F$-root square mean labeling of graphs obtained from paths," International Journal of Mathematical Combinatorics, vol. 2, pp. 92-104, 2017.

[11] S. Arockiaraj, A. Durai Baskar, and A. Rajesh Kannan, "F-root square mean labeling of some ladder related graphs," Global Journal of Pure and Applied Mathematics, vol. 13, no. 2, pp. 281-289, 2017.

[12] A. Durai Baskar and S. Arockiaraj, "C-geometric mean labeling of some ladder graphs," International Journal of Mathematical Combinatorics, vol. 3, pp. 125-140, 2018.

[13] S. Dafik, R. Fitriana Eka, and L. Sya' Diyah, "Super antimagicness of triangular book and diamond ladder graphs," in Proceeding of Indoms International Conference on Mathematics and Its Application, Yogyakarta, Indonesia, November 2013.

[14] A. Durai Baskar, "Logarithmic mean labeling of some ladder graphs," Applications and Applied Mathematics, vol. 15, no. 1, pp. 296-313, 2020. 
[15] A. Rajesh Kannan, P. Manivannan, and R. Rathajeyalakshmi, "Exponential meanness of graphs obtained from some graph operations," Journal of Advanced Research in Dynamical and Control Systems, vol. 10, no. 15, pp. 525-529, 2018.

[16] A. Ahmed and R. Marinescu-Ghemeci, "Radio labeling of some ladder related graphs," Math Reports, vol. 19, no. 69, pp. 107-119, 2017.

[17] M. Zeeneldeen, "Edge $\delta$-graceful labeling for some cyclic related graphs," Advances in Mathematical Physics, vol. 2020, pp. 1-8, Article ID 6273245, 2020.

[18] L. Ratnasari and Y. Susanti, "Total edge irregularity strength of ladder-related graphs," Asian-European Journal of Mathematics, vol. 13, no. 4, Article ID 2050072, 2020.

[19] F. Ashraf, M. Baca, A. Semanicova-Fenovcikova, and M. Kamran Siddiqu, "On H-irregularity strength of ladders and fan graphs," AKCE International Journal of Graphs and Combinatorics, vol. 17, 2019 In press.

[20] M. Seoud and M. Salim, "Further results on edge-odd graceful graphs," Turkish Journal of Mathematics, vol. 40, no. 3, pp. 647-656, 2016.

[21] E. M. Badr, M. I. Moussa, and K. Kathiresan, "Crown graphs and subdivision of ladders are odd graceful," International Journal of Computer Mathematics, vol. 88, no. 17, pp. 35703576, 2011.

[22] E. Y. Kurniawati, I. H. Agustin, Dafik, and Marsidi, "On the super edge local antimagic total labeling of related ladder graph," Journal of Physics: Conference Series, vol. 1465, no. 1, Article ID 012027, 2020.

[23] M. I. Moussa and E. M. Badr, "Ladder and subdivision of ladder graphs with pendent edges are odd graceful," International Journal on Applications of Graph Theory in Wireless Ad Hoc Networks and Sensor Networks, vol. 8, no. 1, pp. 1-8, 2016.

[24] A. A. Elsonbaty and S. N. Daoud, "Edge even graceful labeling of cylinder grid graph," Symmetry, vol. 11, no. 4, pp. 1-30, 2019.

[25] G. Muhiuddin, N. Sridharan, D. Al-Kadi, S. Amutha, and M. E. Elnair, "Reinforcement number of a graph with respect to half-domination," Journal of Mathematics, vol. 2021, Article ID 6689816, 7 pages, 2021.

[26] G. Muhiuddin, A. M. Alanazi, A. R. Kannan, and V. Govindan, "Preservation of the classical meanness property of some graphs based on line graph operation," Journal of Mathematics, vol. 2021, Article ID 4068265, 10 pages, 2021.

[27] G. Muhiuddin, M. M. Takallo, Y. B. Jun, and R. A. Borzooei, "Cubic graphs and their application to a traffic flow problem," International Journal of Computational Intelligence Systems, vol. 13, no. 1, pp. 1265-1280, 2020.

[28] H. Rashmanlou, G. Muhiuddin, Sk Amanathulla, F. Mofidnakhaei, and M. Pal, "A study on cubic graphs with novel application," Journal of Intelligent \& Fuzzy Systems, vol. 40, no. 1, pp. 89-101, 2021.

[29] S. S. Hussain, R. J. Hussain, and G. Muhiuddin, "Neutrosophic vague line graphs," Neutrosophic Sets and Systems, vol. 36, pp. 121-130, 2020.

[30] T. Pramanik, G. Muhiuddin, A. M. Alanazi, and M. Pal, "An extension of fuzzy competition graph and its uses in manufacturing industries," Mathematics, vol. 8, p. 1008, 2020.

[31] S. Samanta, G. Muhiuddin, A. M. Alanazi, and K. Das, "Mathematical approach on representation of competitions: competition cluster hypergraphs," Mathematical Problems in Engineering, vol. 2020, p. 10, Article ID 2517415, 2020. 